\title{
Ejaculatory disorders after prostatic artery embolization: a reassessment of two prospective clinical trials
}

\author{
Gautier Müllhaupt ${ }^{1}$ (D) Lukas Hechelhammer $^{2} \cdot$ Pierre-André Diener $^{4} \cdot$ Daniel S. Engeler $^{1} \cdot$ Sabine Güsewell $^{3}$. \\ Hans-Peter Schmid ${ }^{1} \cdot$ Livio Mordasini $^{1} \cdot$ Dominik Abt $^{1}$
}

Received: 18 May 2019 / Accepted: 21 November 2019 / Published online: 7 December 2019

(c) The Author(s) 2019

\begin{abstract}
Purpose This study aims to specify and explain the previous findings of unexpectedly high rates of ejaculatory disorders, i.e. 56\%, found after prostatic artery embolization (PAE) in a randomized controlled trial comparing safety and efficacy of PAE and transurethral resection of the prostate (TURP).

Patients and methods Case report forms of the randomized controlled trial were analyzed to specify the grade of postoperative ejaculatory dysfunction 3 months postoperatively.

In addition, study participants with assessable ejaculation were asked to complete the four-item Male Sexual Health Questionnaire-Ejaculation Dysfunction Short Form (MSHQ-EjD) referring to their ejaculatory function at present, as well as before treatment and 3 months after. Potential explanations for ejaculatory disorders after PAE were derived from histological examination of five radical prostatectomy specimens of patients that underwent PAE 6 weeks before radical prostatectomy within a proof-of-concept trial at the study site, St. Gallen Cantonal Hospital. An experienced uropathologist systematically examined the whole-gland embedded tissue with focus on structures that are involved into ejaculation.

Results While patients after TURP predominantly suffered from anejaculation (52\%), diminished ejaculation was found more often after PAE (40\%). Significantly higher MSHQ-EjD scores were found 3 months after PAE and at a median follow-up of 31 months.

Histological examination showed marked changes of structures involved into ejaculation (e.g., prostatic glands, seminal vesicles, ejaculatory ducts) after PAE.

Conclusion Although anejaculation occurs less frequently after PAE (16\%) compared to TURP (52\%), patients have to be informed about the relevant risk of ejaculatory disorders, especially diminished ejaculation.
\end{abstract}

Keywords Anejaculation $\cdot$ Benign prostatic hyperplasia $\cdot$ Diminished ejaculation $\cdot$ Ejaculatory disorders $\cdot$ Prostatic artery embolization $\cdot$ Retrograde ejaculation

Gautier Müllhaupt

gautier.muellhaupt@kssg.ch

1 Department of Urology, St. Gallen Cantonal Hospital, Rorschacherstrasse 95, 9007 St. Gallen, Switzerland

2 Department of Radiology and Nuclear Medicine, St. Gallen Cantonal Hospital, St. Gallen, Switzerland

3 Clinical Trials Unit, St. Gallen Cantonal Hospital, St. Gallen, Switzerland

4 Department of Pathology, St. Gallen Cantonal Hospital, St. Gallen, Switzerland

\section{Introduction}

Preservation of ejaculatory function is an important issue for many patients undergoing surgery for lower urinary tract symptoms associated with benign prostatic hyperplasia (BPH/LUTS). Reliable preservation of ejaculation is, however, hardly possible with most surgical options, especially if a clear relief of bladder outlet obstruction is anticipated [1].

Prostatic artery embolization was considered to have no influence on ejaculation until recently [2]. In 2018, Ray et al. reported on ejaculatory disorders (referred to as "retrograde ejaculation") in $24.1 \%$ of patients after PAE compared to $47.5 \%$ after transurethral resection of the prostate (TURP) in a matched-pair analysis [3]. In our randomized controlled 
trial comparing PAE and TURP [4], changes in ejaculatory function were assessed according to the CTCAE classification [5] (i.e., grade 1: "diminished", grade 2: "anejaculation or retrograde ejaculation"). Both grades summarized occurred in $56 \%(14 / 25)$ and $84 \%(21 / 25)$ of patients in whom ejaculation was assessable after PAE and TURP, respectively. These discrepancies compared to previous studies led to confusions and uncertainties in patient counseling.

The present study aims to specify and explain our previous findings by providing more precise data on patients' ejaculatory function. In addition, we examined post-PAE prostatectomy specimens derived from a proof-of-concept study [6] considering structures involved into ejaculation to assess potential underlying mechanisms of ejaculatory disorders after PAE.

\section{Patients and methods}

Case report forms of the randomized controlled trial were analyzed to specify the grade of postoperative ejaculatory dysfunction (i.e., grade 1: diminished vs. grade 2: anejaculation or retrograde ejaculation) 3 months postoperatively.

In addition, study participants with assessable ejaculation were asked to complete the four-item Male Sexual Health Questionnaire-Ejaculation Dysfunction Short Form (MSHQEjD) [7], a validated and abridged version of the 25-item Male Sexual Health Questionnaire (MSHQ) for assessing ejaculatory dysfunction, referring to their ejaculatory function at present, as well as (retrospectively) before treatment and 3 months later. Wilcoxon rank sum tests were used to determine the significance of differences in MSHQ-EjD scores between PAE and TURP.

Potential explanations for ejaculatory disorders after PAE were derived from histological examination of five radical prostatectomy specimens of patients that underwent PAE 6 weeks before radical prostatectomy for localized prostate cancer within a proof-of-concept trial at the study site St. Gallen Cantonal Hospital [6]. An experienced uropathologist (PAD) systematically examined the whole-gland embedded tissue with focus on structures that are involved into ejaculation (i.e., prostatic glands central, peripheral, and adjacent to verumontanum, ejaculatory ducts, seminal vesicles). Tissue changes (i.e., necrosis, fibrosis, hemorrhage) were categorized into none, $<10 \%, 10-50 \%$, and $>50 \%$ and occurrence of particles was categorized to none, few, moderate, and abundant.

The study was approved by the local ethics committee (EKSG 14/004 and BASEC PB_2016-02294).
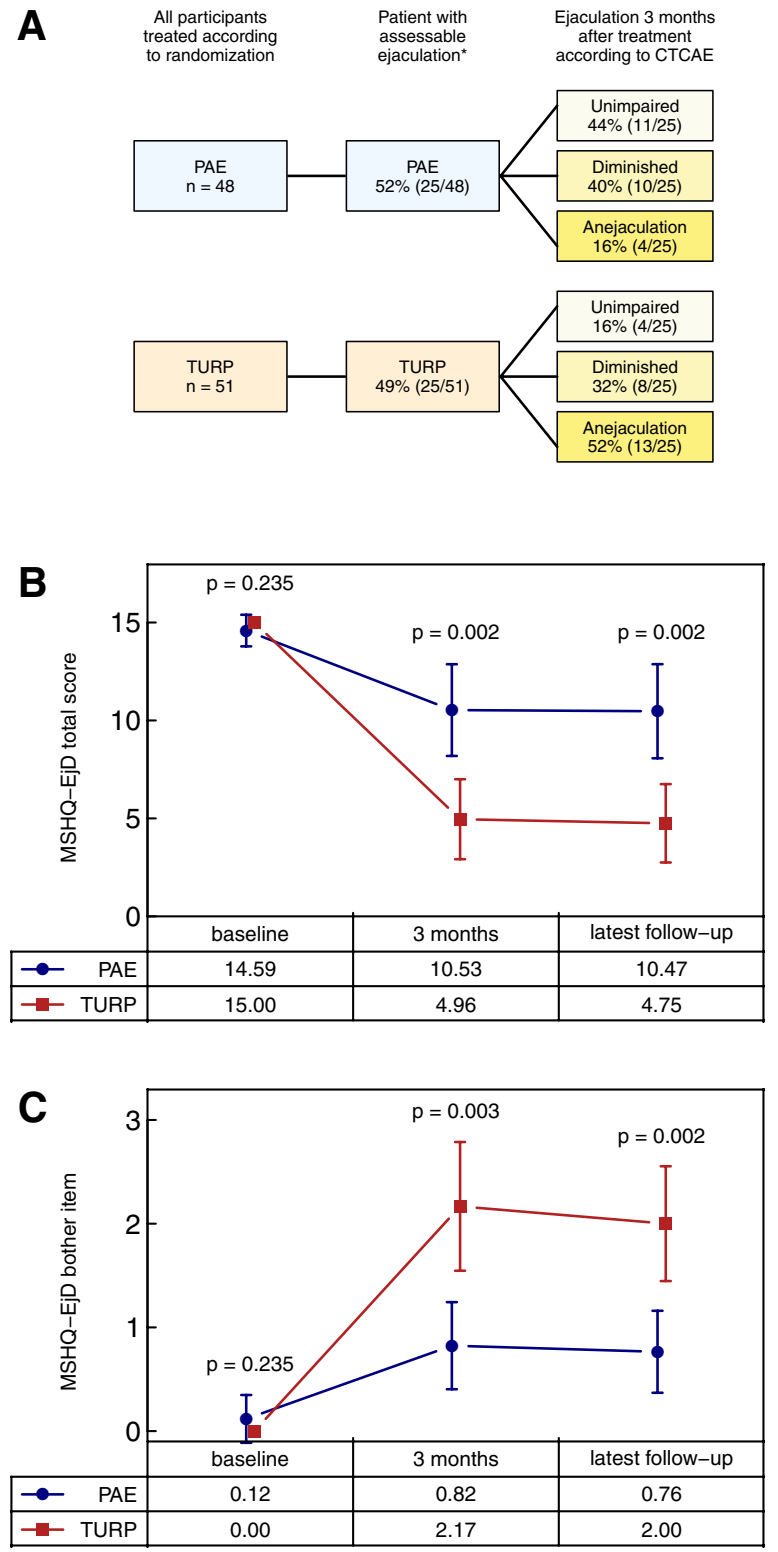

Fig. 1 Postoperative ejaculatory disorders assessed according to CTCAE [5] (a), Male Sexual Health Questionnaire-Ejaculation Dysfunction Short Form (MSHQ-EjD) ejaculatory function total score (questions 1-3, possible range 0-15) (b) and MSHQ-EjD ejaculatory bother item (question 4, possible range 0-5) (c) [bars show means and $95 \% \mathrm{CI}$, numbers are means, and $p$ values from Wilcoxon rank sum tests indicate the significance of differences between PAE and TURP. Asterisk: ejaculation was considered as not assessable in patients with complete erectile dysfunction and in patients reporting to have no sexual stimulation at all; note that MSHQ-EjD was completed retrospectively by the patients for baseline and 3 month followup. Latest follow-up was median 31 months (17-58)] 


\section{Results}

Based on case report forms of the original trial, ejaculatory disorders occurred more frequently and were more pronounced after TURP compared to PAE (Fig. 1a). However, after PAE, $16 \%$ and $40 \%$ of patients reported on anejaculation or diminished ejaculation, respectively. Baseline characteristics of the patients with assessable ejaculation 12 weeks after intervention are shown in Table 1.

Forty-one patients were available for the MSHQ-EjDbased survey. Ejaculatory function at baseline was rated as unimpaired by nearly all patients of both groups (Fig. 1b). While, a deterioration of ejaculatory function occurred after PAE and TURP, it was significantly more pronounced after TURP after 3 months and after a median follow-up for 31 months (range 17-58). In accordance, patients were significantly more bothered by ejaculatory disorders after TURP. Thus, the mean rating of bothersomeness after 3 months was between 2 and 3 ("a little bothered" to "moderately bothered") after TURP compared to less than 1 ("not at all bothered") after PAE (Fig. 1c).

Histological examination of post-PAE radical prostatectomy specimens revealed extensive fibrosis, necrosis, and hemorrhage. These changes were most distinct at the central gland, but also occurred in all other structures relevant for ejaculatory function in all of the five patients assessed (Table 2, Fig. 2).

\section{Discussion}

This study elucidates the so far sparse and inconsistent data on ejaculatory function after PAE. Although anejaculation occurs less frequently after PAE compared to TURP, patients have to be informed about a substantial risk of ejaculatory disorders, especially diminished ejaculation.

According to the results of our analysis, median bothersomeness of ejaculatory deterioration was rather low in the setting of the original randomized controlled trial. However, preservation of ejaculatory function represents a widely underestimated issue for patients undergoing surgical treatment of BPH/LUTS $[8,9]$ and, therefore, our results provide important data for patient counseling.

Fibrosis and necrosis of structures involved into ejaculation (Table 1) were identified as possible underlying mechanisms. Considering the fact that these changes were found in all of the structures of each of the patients, absence of ejaculatory disorders in previous trials [2] seems to be caused by underreporting.

Compared to our study, clearly lower rates of ejaculatory disorders have been reported for other minimally invasive treatment options (e.g., Urolift $^{\circledR}$, Rezum $^{\circledR}$ ) [1]. However, as direct comparisons of PAE with other minimally invasive treatment options are not available and ejaculation preservation rates are highly dependent on the type of assessment performed within a study, further data seem to be mandatory to determine the best treatment for patients aiming at preservation of ejaculatory function.

Strengths of our assessment include the availability of post-PAE radical prostatectomy specimens that allowed for a detailed microscopic assessment and the prospective assessment of ejaculatory changes according to the CTCAE classification within the randomized controlled trial. However, the MSHQ-EjD was not implemented into the study and, therefore, was assessed retrospectively for the baseline and 3 month follow-up visit. As a large proportion of the patients took alpha-blockers, 5-alphareductase inhibitors, or a combination of both at the time of the baseline visit (Table 1); baseline MSHQ-EjD-scores seem to be surprisingly high. Thus, ejaculatory function
Table 1 Baseline characteristics of the patients with assessable ejaculation 12 weeks after intervention.

\begin{tabular}{lll}
\hline Characteristic $^{\mathrm{a}}$ & PAE $(N=25)$ & TURP $(N=25)$ \\
\hline Age, years & $62.9 \pm 7.5$ & $63.1 \pm 9.0$ \\
Body-mass-index, $\mathrm{kg} \mathrm{m}^{-2}$ & $25.8 \pm 3.5$ & $26.8 \pm 3.2$ \\
Charlson comorbidity index & $3.2 \pm 1.2$ & $4.0 \pm 2.1$ \\
Prostate volume (transabdominal ultrasound), $\mathrm{mL}$ & $50.6 \pm 16.3$ & $52.1 \pm 20.1$ \\
Prostate volume (magnetic resonance imaging), $\mathrm{mL}$ & $49.8 \pm 31.8$ & $59.7 \pm 35.3$ \\
Medical treatment of LUTS prior to surgery, no. $(\%)$ & & \\
5-Alpha-reductase inhibitors alone & $0(0 \%)$ & $0(0 \%)$ \\
Alpha ${ }_{1}$-adrenergic receptor antagonists & $12(48 \%)$ & $12(48 \%)$ \\
Combination of the two above & $7(28 \%)$ & $4(16 \%)$ \\
Total patients with drug treatment & $19(76 \%)$ & $16(64 \%)$ \\
Indwelling urethral catheter at baseline, no. $(\%)$ & $4(16 \%)$ & $3(12 \%)$ \\
\hline
\end{tabular}

${ }^{a}$ Numeric data are summarized as mean \pm standard deviation and categorical data as number and percentage 
Table 2 Histological findings of structures involved to ejaculation in five patients undergoing radical prostatectomy 6 weeks after PAE (necrosis/ fibrosis/hemorrhage: 0 : none, $+<10 \%,++10-50 \%,+++>50 \%$; occurrence of particles: 0 : none, + few, ++ moderate, +++ abundant)

\begin{tabular}{|c|c|c|c|c|c|c|}
\hline \multirow[b]{2}{*}{ Localization } & \multirow[b]{2}{*}{ Findings } & \multicolumn{5}{|c|}{ Patient no. } \\
\hline & & 1 & 2 & 3 & 4 & 5 \\
\hline \multirow[t]{2}{*}{ Prostate-central gland } & Necrosis/fibrosis/hemorrhage & +++ & +++ & +++ & $\begin{array}{l}+++(\text { anterior }) \\
++(\text { posterior })\end{array}$ & $\begin{array}{l}+++(\text { anterior }) \\
++(\text { posterior })\end{array}$ \\
\hline & Particles & ++ & +++ & ++ & ++ & $\begin{array}{l}+++(\text { anterior }) \\
++(\text { posterior })\end{array}$ \\
\hline \multirow[t]{3}{*}{ Prostate-peripheral gland } & Necrosis/fibrosis/hemorrhage & + & $\begin{array}{l}++(\text { anterior }) \\
+(\text { posterior })\end{array}$ & $\begin{array}{l}+++(\text { anterior }) \\
++(\text { posterior })\end{array}$ & $\begin{array}{l}+++(\text { anterior }) \\
++(\text { posterior })\end{array}$ & ++ \\
\hline & Particles & + & $\begin{array}{l}+++(\text { anterior }) \\
+(\text { posterior })\end{array}$ & +++ & $\begin{array}{l}++(\text { anterior }) \\
+(\text { posterior })\end{array}$ & +++ \\
\hline & Particles in adjacent soft tissue & ++ & $\begin{array}{l}+++(\text { anterior }) \\
++(\text { posterior })\end{array}$ & +++ & $\begin{array}{l}+++(\text { anterior }) \\
++(\text { posterior })\end{array}$ & $\begin{array}{l}+(\text { anterior }) \\
+++(\text { posterior })\end{array}$ \\
\hline \multirow{2}{*}{$\begin{array}{l}\text { Prostate-adjacent to verumon- } \\
\text { tanum }\end{array}$} & Necrosis/fibrosis/hemorrhage & + & + & ++ & ++ & ++ \\
\hline & Particles & + & +++ & + & ++ & ++ \\
\hline \multirow[t]{3}{*}{ Ejaculatory ducts } & Necrosis/fibrosis/hemorrhage & ++ & ++ & ++ & +++ & ++ \\
\hline & Particles & + & + & 0 & ++ & + \\
\hline & Particles in adjacent soft tissue & ++ & + & ++ & 0 & 0 \\
\hline \multirow[t]{3}{*}{ Seminal vesicles } & Necrosis/fibrosis/hemorrhage & +++ & ++ & +++ & +++ & ++ \\
\hline & Particles & + & + & + & ++ & +++ \\
\hline & Particles in adjacent soft tissue & ++ & + & ++ & +++ & ++ \\
\hline
\end{tabular}
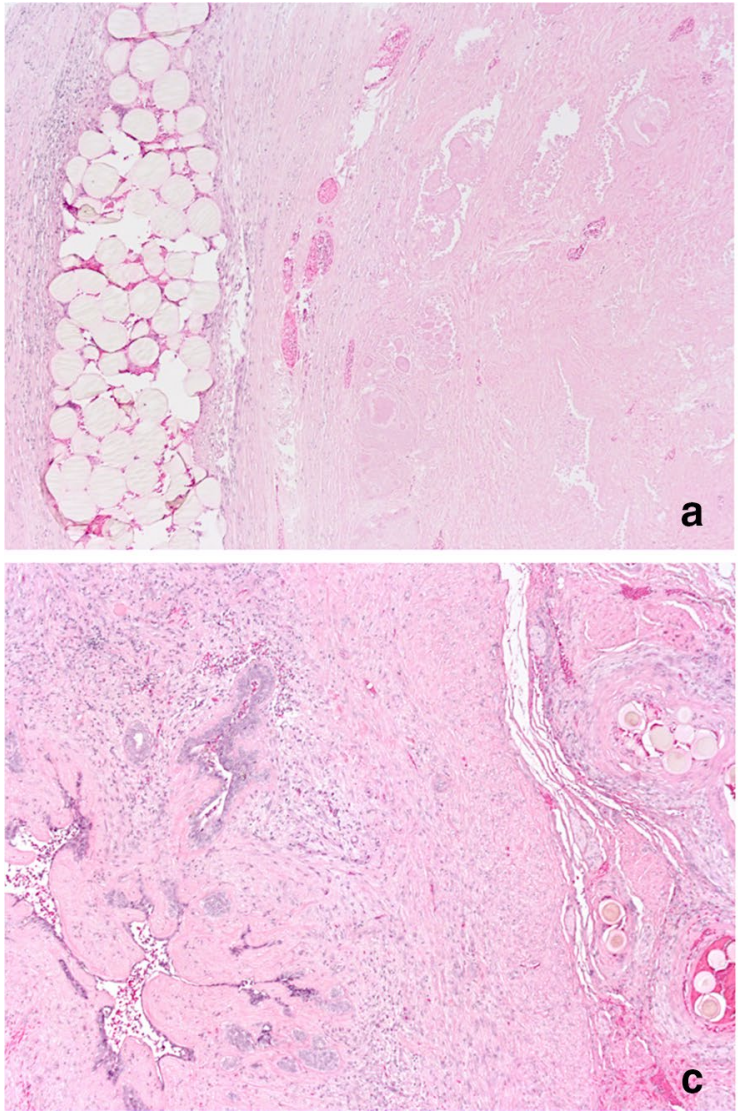

Fig. 2 Histological findings in patients undergoing radical prostatectomy 6 weeks after prostatic artery embolization. Selected pictures (HE staining) show extensive necrosis next to embolization particles

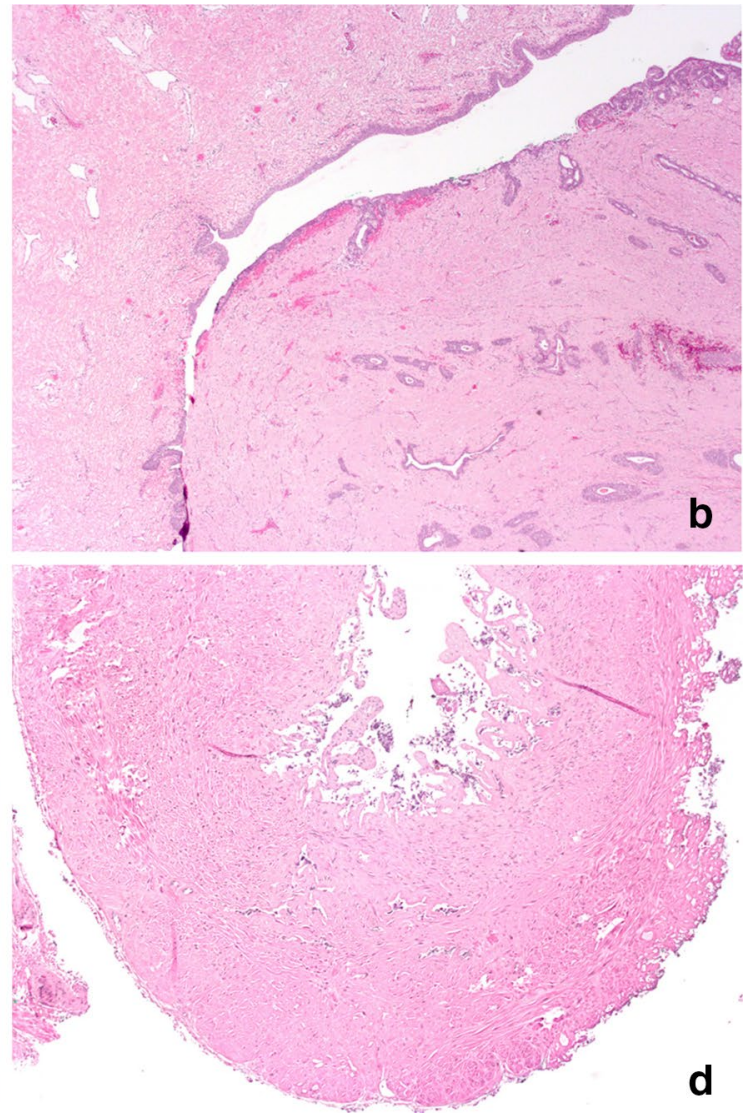

in the central prostatic gland $(\mathbf{a}, \times 50)$, extensive fibrosis around the verumontanum $(\mathbf{b}, \times 25)$ and mucosal necrosis and atrophy of the seminal vesicles $(\mathbf{c}, \times 50)$ and ejaculatory duct $(\mathbf{d}, \times 50)$ 
before treatment might has been rated as normal condition or overestimated. Moreover, $100 \mu \mathrm{m}$ microspheres were used in the proof-of-concept study, from which we obtained tissue samples and histological changes might differ with varying particle sizes.

As ejaculatory function was not in the limelight of our randomized trial, TURP was not performed in an ejaculatory sparing technique, which has to be considered when comparing the results between PAE and TURP. Thus, a significant reduction of ejaculatory disorders has been demonstrated after ejaculation sparing transurethral surgery [1].

\section{Conclusions}

Ejaculatory disorders occur in about $56 \%$ of patients undergoing PAE and might result from degenerative changes of anatomical structures involved into ejaculation after PAE. While the majority of these patients develop diminished ejaculation (40\%), anejaculation can also occur (16\%).

Author contributions Protocol/project development: GM, DA. Data collection or management: GM, DA, LH, P-AD. Data analysis: GM, DA, SG, P-AD. Manuscript writing/editing: GM, DA. Critical revision of the manuscript: H-PS, DSE, LM, LH. Statistical analysis: SG, GM. Supervision: DA.

Funding The studies were funded by a grant of the research committee of the hospital (14/08 and 15/16). There was no commercial support.

\section{Compliance with ethical standards}

Conflict of interest Boston Scientific (Natick, MA, USA) provided Embozene microspheres for the patients in the proof-of-concept study assessing PAE in localized prostate cancer free of charge. The company did not influence design, conduct, and analysis of the study. DA: Research support (institutional): Olympus, Boston Scientific, Advisory role (institutional): Janssen, Travel support (institutional): Janssen, Debiopharm. GM: Travel support: Debiopharm.

Informed consent Informed consent was obtained from all individual participants included in the study.

Ethical approval All procedures performed in studies involving human participants were in accordance with the ethical standards of the institutional and/or national research committee and with the 1964 Helsinki Declaration and its later amendments or comparable ethical standards.

Open Access This article is distributed under the terms of the Creative Commons Attribution 4.0 International License (http://creativeco mmons.org/licenses/by/4.0/), which permits unrestricted use, distribution, and reproduction in any medium, provided you give appropriate credit to the original author(s) and the source, provide a link to the Creative Commons license, and indicate if changes were made.

\section{References}

1. Lebdai S, Chevrot A, Doizi S, Pradere B, Delongchamps NB, Benchikh A, Cornu JN, Della Negra E, Fourmarier M, Misrai V, Theveniaud PE, Descazeaud A, Robert G, CTMH-AFU Group (2019) Do patients have to choose between ejaculation and miction? A systematic review about ejaculation preservation technics for benign prostatic obstruction surgical treatment. World J Urol. 37(2):299-308. https://doi.org/10.1007/s00345-018-2368-6(epub 2018 Jul 2)

2. Shim SR, Kanhai KJ, Ko YM, Kim JH (2017) Efficacy and safety of prostatic arterial embolization: systematic review with metaanalysis and meta-regression. J Urol 197(2):465-479. https://doi. org/10.1016/j.juro.2016.08.100

3. Ray AF, Powell J, Speakman MJ, Longford NT, DasGupta R, Bryant T, Modi S, Dyer J, Harris M, Carolan-Rees G, Hacking N (2018) Efficacy and safety of prostate artery embolization for benign prostatic hyperplasia: an observational study and propensity-matched comparison with transurethral resection of the prostate (the UK-ROPE study). BJU Int 122(2):270-282. https://doi. org/10.1111/bju.14249

4. Abt D, Hechelhammer L, Mullhaupt G, Markart S, Gusewell S, Kessler TM, Schmid HP, Engeler DS, Mordasini L (2018) Comparison of prostatic artery embolisation (PAE) versus transurethral resection of the prostate (TURP) for benign prostatic hyperplasia: randomised, open label, non-inferiority trial. BMJ 361:k2338. https://doi.org/10.1136/bmj.k2338

5. National Cancer Institute (NCI) common terminology criteria for adverse events (CTCAE) version 4.0. https://evs.nci.nih.gov/ftp1/ CTCAE/CTCAE_4.03/CTCAE_4.03_2010-06-14_QuickRefer ence $5 \times 7$.pdf

6. Mordasini L, Hechelhammer L, Diener PA, Diebold J, Mattei A, Engeler D, Mullhaupt G, Kim SK, Schmid HP, Abt D (2018) Prostatic artery embolization in the treatment of localized prostate cancer: a bicentric prospective proof-of-concept study of 12 patients. J Vasc Interv Radiol 29(5):589-597. https://doi.org/10.1016/j. jvir.2018.01.766

7. Rosen RC, Catania JA, Althof SE, Pollack LM, O'Leary M, Seftel AD, Coon DW (2007) Development and validation of four-item version of Male Sexual Health Questionnaire to assess ejaculatory dysfunction. Urology 69(5):805-809. https://doi.org/10.1016/j. urology.2007.02.036

8. Feldman HA, Goldstein I, Hatzichristou DG, Krane RJ, McKinlay JB (1994) Impotence and its medical and psychosocial correlates: results of the Massachusetts Male Aging Study. J Urol 151(1):54-61

9. Kim JK, Cho MC, Son H, Ku JH, Oh SJ, Paick JS (2017) Patient perception of ejaculatory volume reduction after holmium laser enucleation of the prostate (HoLEP). Urology 99:142-147. https ://doi.org/10.1016/j.urology.2016.09.037

Publisher's Note Springer Nature remains neutral with regard to jurisdictional claims in published maps and institutional affiliations. 